\title{
INTEGRATED THERMAL CONDUCTIVITY VACUUM SENSOR
}

\author{
Erno H. Klaassen and Gregory T.A. Kovacs \\ Center for Integrated Systems, CIS-X 202 \\ Stanford University, Stanford, CA 94305-4075
}

\begin{abstract}
A thermal conductivity vacuum sensor with a novel on-chip control system is presented. The sensing structure is a small circuit suspended over a cavity etched in the substrate. An integrated control system maintains a constant temperature difference between this thermally isolated sensor and the substrate. The control loop allows for a digitally adjustable operating temperature of the sensing structure. The range of the vacuum sensor extends from $0.8 \mathrm{~Pa}(6 \mathrm{mTorr})$ to approximately $9.2 \times 10^{4} \mathrm{~Pa}$ (690 Torr). The system was fabricated in a completely unmodified foundry CMOS process, dissipates only $5 \mathrm{~mW}$ of power, and occupies an area of approximately $0.3 \mathrm{~mm}^{2}$.
\end{abstract}

\section{INTRODUCTION}

Thermal conductivity gauges include the Pirani, thermocouple, and thermistor gauges. These devices measure pressure indirectly by sensing changes in the thermal conductivity of the ambient gases [1]. The thermal conductivity of a gas can be expressed as

$$
k=\frac{1}{3} m \lambda n v_{a v e} c_{v}
$$

where $m$ is the molecular mass, $\lambda$ is the mean-free-path, $n$ is the molecular density, $v_{\text {ave }}$ is the average molecular velocity of the molecules, and $c_{v}$ is the specific heat at constant volume of the gas. The thermal conductivity of a gas, as a material property, does not change with pressure, since the pressure dependence of $\lambda$ and $n$ cancels. Thermal conductivity vacuum gauges therefore must limit the mean-free-path by some geometrical constraint.

Thermal vacuum sensors, like other thermally based instrumentation, can benefit from miniaturization. The reduction of parasitic thermal losses and thermal time constants can yield lower power consumption and faster response. These benefits, as well as the prospect of providing single-chip sensing systems have led to the development of a number of micromachined vacuum sensors over recent years $[2,3,4,5,6,7]$. Only some of these sensors have on-chip circuitry, and most are made with a custom fabrication process.

The fully integrated thermal vacuum sensor presented here is fabricated using post-processing methods on an unmodified foundry CMOS process [8]. The sensor measures the pressuredependent thermal losses from a hot element, which consists of an area of single crystal silicon suspended by silicon dioxide support beams. The device is fabricated with an electrochemical etch in silicon-doped tetramethyl ammonium hydroxide (TMAH). Figure 1 shows a cross section of the undercutting etch that thermally isolates the circuitry. The details of the fabrication process can be found elsewhere $[\mathbf{9 , 1 0}]$. Elements with high thermal isolation, yet with relatively small size, can be fabricated in this fashion. In addition, the suspended silicon can contain efficient heating and temperature sensing elements.

The suspended element is maintained at constant temperature relative to the substrate. The power necessary to do so is a function of thermal conduction through the support beams, but also has a convective component. When the mean free path of the molecules is dictated by the spacing between the silicon and the etched cavity, the convective component becomes a measure of air pressure, as shown in Eqn. 1.

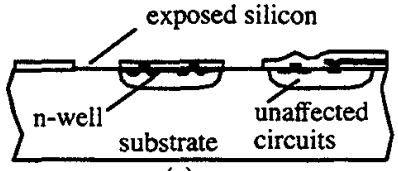

(a)

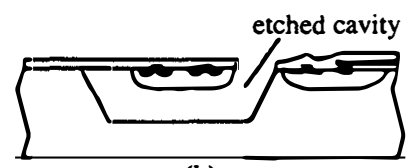

(b)
Figure 1. (a) Cross section of a vacuum sensor upon completion of the CMOS process. (b) Cross section of a completed vacuum sensor after the post-processing etch. The suspended silicon contains a PMOS heater and a diode temperature sensor.

\section{SENSING ELEMENT DESIGN}

An electrical analog of the thermal behavior of a thermally isolated sensor is shown in Figure 2. The thermal conductances are modeled as electrical conductors, heat dissipation is depicted by a current source, and voltages inside this circuit reflect temperature. The reference, indicated as an electrical ground, is the temperature of the silicon substrate around the sensor. The conductive losses are split into two components. The first is the unchanging conductive loss through the support beams of the element, which is dominated by the $0.5 \mu \mathrm{m}$ thick aluminum traces in the beams. To minimize this parasitic element, the beams are made long, and the aluminum traces are made minimum width ( 3 $\mu \mathrm{m})$. The other component is a pressure dependent thermal conductance through the air between the heated circuitry and the silicon substrate. In order to maximize the ratio of the two conductors, the heated element was placed close to the corner of the etched pit, so that the dominant conduction through the gas occurs between the silicon element and the (111) side-wall.

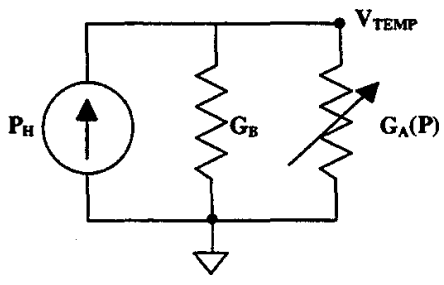

Figure 2. Electrical analog of the thermal behavior of the sensing element. The heat dissipated in the suspended circuit is modeled as a current source, while the temperature is denoted as the nodal temperature $V_{T E M P}$. The reference point in the circuit is the substrate temperature.

Figure 3 shows a top view of a vacuum sensing element. Devices were fabricated with beam lengths of $240 \mu \mathrm{m}$ and 340 $\mu \mathrm{m}$. The heated element requires a temperature sensor and a heater. In previous micromachined references, these functions were often performed with a single resistor $[3,5,6,7]$. In this technology, however, separate devices can be used for each of 
these transduction operations between the electrical and thermal signal domains. A diode-connected bipolar transistor was used as the temperature sensor. When forward-biased with a constant current, the voltage across these devices has a temperature sensitivity of $-2 \mathrm{mV} / \mathrm{K}$. A polysilicon or diffused resistor could be used as a heater, but since the power dissipated in a resistor has a square-law dependence on voltage across or current through the device, special circuitry would be necessary to provide an output proportional to the conductive losses in the sensor. The on-chip feedback amplifier would also need to drive a resistive load. A better solution is to use a PMOS heating transistor. The power dissipated in such a device is proportional to source-drain current for a constant source-drain voltage and the amplifier only needs to drive the gate capacitance of the heater.

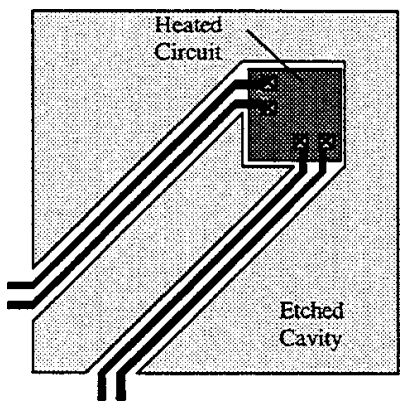

Figure 3. Illustration showing a top view of a sensing element. The circuitry at the tip of the device is heated.

\section{SYSTEM DESIGN}

Figure 4 shows a simplified schematic of the vacuum sensor system. The shaded devices are located on the sensing element. The output voltage between the two forward-biased diodes is proportional to the temperature difference between the sensor and the substrate. This temperature difference must be maintained at a constant value. The source-drain current through the PMOS transistor is then proportional to the power dissipated to maintain this temperature set-point.

The set-point could be implemented by adjusting the ratio of the current densities of the two diodes. This creates a voltage offset between the two diodes that is driven to zero by the feedback amplifier, thus generating a temperature difference between the sensor and the substrate. The voltage between two diodes (at equal temperatures) is given by

$$
v_{O}=v_{t} \ln \left(\frac{I_{D 1}}{I_{S 1}}\right)-v_{t} \ln \left(\frac{I_{D 2}}{I_{S 2}}\right)=v_{t} \ln \left(\frac{n}{m}\right)
$$

where $v_{t}$ is the thermal voltage $(\mathrm{kT} / \mathrm{q}), I_{D}$ is the current through the diodes, $I_{S}$ is the reverse saturation current, $n$ is the ratio of $I_{D I}$ to $l_{D 2}$, and $m$ is the junction area ratio of the two devices. The offset cannot be varied over a very wide range, due to the natural logarithm in the expression. Another problem is that the voltage is proportional to absolute temperature, which makes the operating point of the element a strong function of ambient temperature.

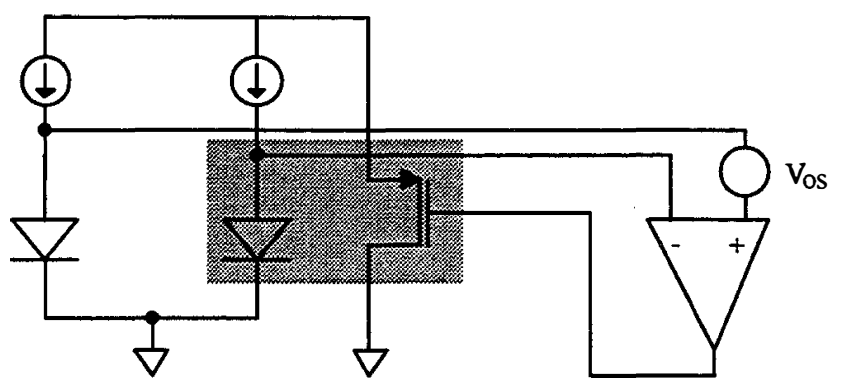

Figure 4. Simplified schematic of the vacuum sensor system. The shaded area is the thermally isolated circuit. The output voltage between the two forward biased diodes is proportional to the temperature difference between the sensor and the substrate.

A better way to set the operating temperature of the element is to introduce an offset inside the feedback amplifier. One way to do this is to effect a threshold voltage difference in the input devices of an operational amplifier. A topology that realizes this is shown in Figure 5.

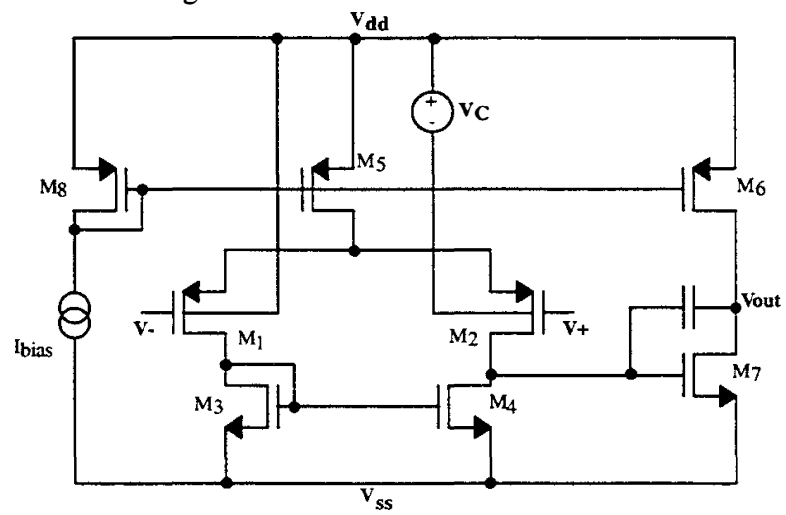

Figure 5. Two-stage CMOS operational amplifier with adjustable offset.

The n-well that contains the non-inverting input transistor $\mathrm{M}_{2}$ is held at a voltage $V_{C}$ below the well containing the other input transistor. The threshold voltage of an MOS transistor is modulated by the body effect, and for a PMOS device can be written as

$$
V_{T}=V_{T 0}-\gamma\left[\sqrt{\left|2 \varphi_{f}\right|-V_{S B}}-\sqrt{\left|2 \varphi_{f}\right|}\right]
$$

where $V_{T O}$ is the threshold voltage without the body effect, $2 \phi_{\mathrm{f}}$ is the surface potential at inversion, $V_{s b}$ is the source-to-bulk voltage, and $\gamma$ is the body effect coefficient. The difference in offsets is

$$
\left|\Delta V_{T}\right|=\gamma\left[\sqrt{\left|2 \varphi_{f}\right|-V_{S B 1}}-\sqrt{\left|2 \varphi_{f}\right|-V_{S B 2}}\right]
$$

With an offset introduced in this fashion, the common-mode rejection of the amplifier is degraded from one with a balanced input pair. The common-to-differential mode rejection ratio (CMRR), however, remains high if the differential gain is sufficiently large. This CMRR rejects the effects of commonmode changes in the temperature sensor output that result from ambient temperature changes.

The offset of the amplifier as a function of the offset control voltage $V_{C}$ was measured and is plotted in Figure 6. 


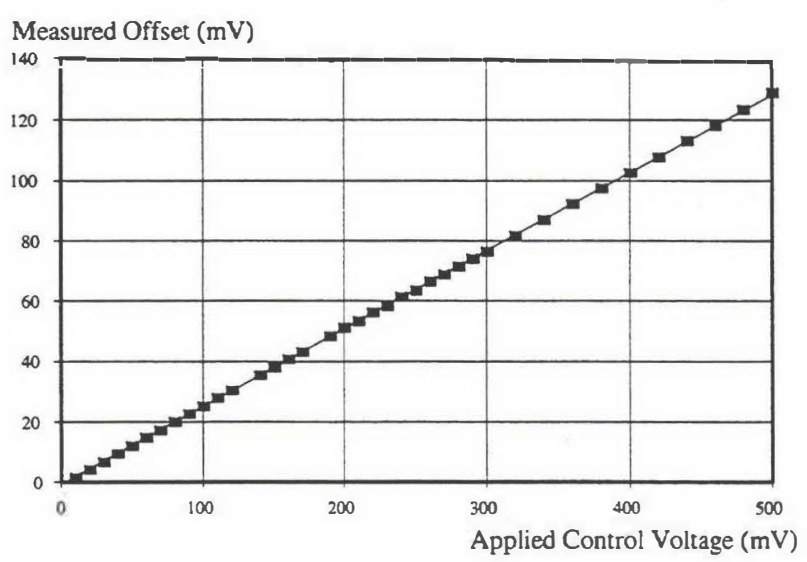

Figure 6. Measured input-referred offset of the amplifier in Figure 5 as a function of the control voltage $V_{C}$.

In order to generate the offset-control voltage, a digital-toanalog converter (DAC) was implemented on-chip. This allows for effective external control of the operating temperature of the sensing element by digital means, such as a microprocessor. The schematic of the DAC is shown in Figure 7. The DAC is based on a current-mirror consisting of bipolar transistors $\mathrm{Q}_{1}$ and $\mathrm{Q}_{2}$. These transistors are made by using a p-type base implant available in the CMOS process. The MOS devices at the emitters of the NPN transistors operate in the linear region, and act as an equivalent resistance. Kirchoff's voltage law around the loop containing the linear MOS transistors and the two bipolars yields (ignoring base currents)

$$
v_{t} \ln \left(\frac{I_{1}}{I_{2}}\right)+I_{1} R_{A}=I_{2} R_{B}
$$

where $I_{1}$ and $I_{2}$ are the collector currents of $\mathrm{Q}_{1}$ and $\mathrm{Q}_{2}$, respectively. $R_{A}$ is the effective resistance of linear MOS device $M_{6}$, and $R_{B}$ is the source-drain resistance of the parallel combination of $M_{7}$ through $M_{12}$. With proper device sizing and biasing, the contribution of the natural logarithm term can be neglected, and the expression above simplifies to

$$
I_{2} \approx \frac{I_{1} R_{A}}{R_{B}}
$$

The current ratio in the mirror is therefore determined by the ratio of the resistances at the emitter nodes. The resistance $R_{B}$ is set by a digital code applied to the binary-weighted set of NMOS transistors $\mathbf{M}_{7}-\mathbf{M}_{12}$.

The entire vacuum sensor system is shown in Figure 8. The power dissipated in the PMOS heater is proportional to the current through this device, which is the current output of the vacuum sensor.

\section{RESULTS}

Figure 9 shows an SEM photograph of an etched vacuum sensor element. The beams on this sensor are $240 \mu \mathrm{m}$ long. The temperature-sensing diode is located at the far comer of the element, and the PMOS heater (two separate devices) is located along the inner edge of the suspended silicon island.

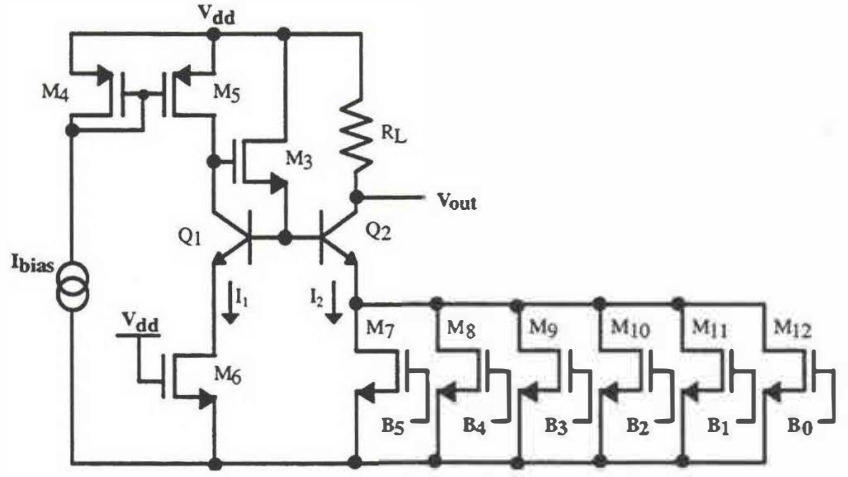

Figure 7. DAC that generates the offset control voltage for the feedback amplifier.

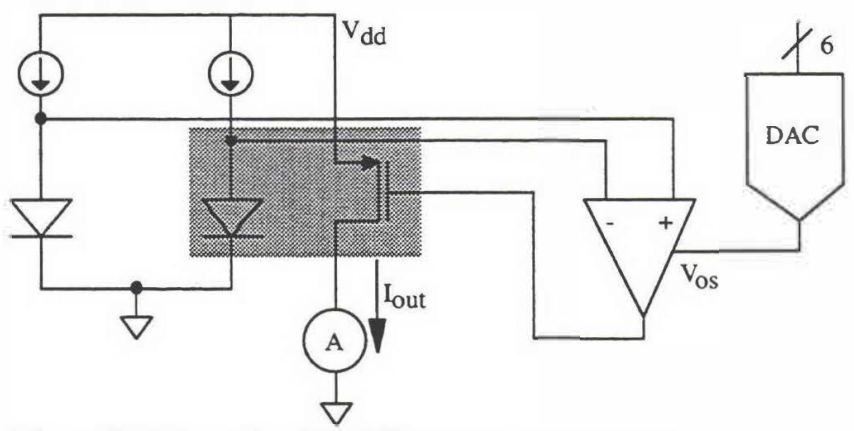

Figure 8. Schematic of the full vacuum sensor system.

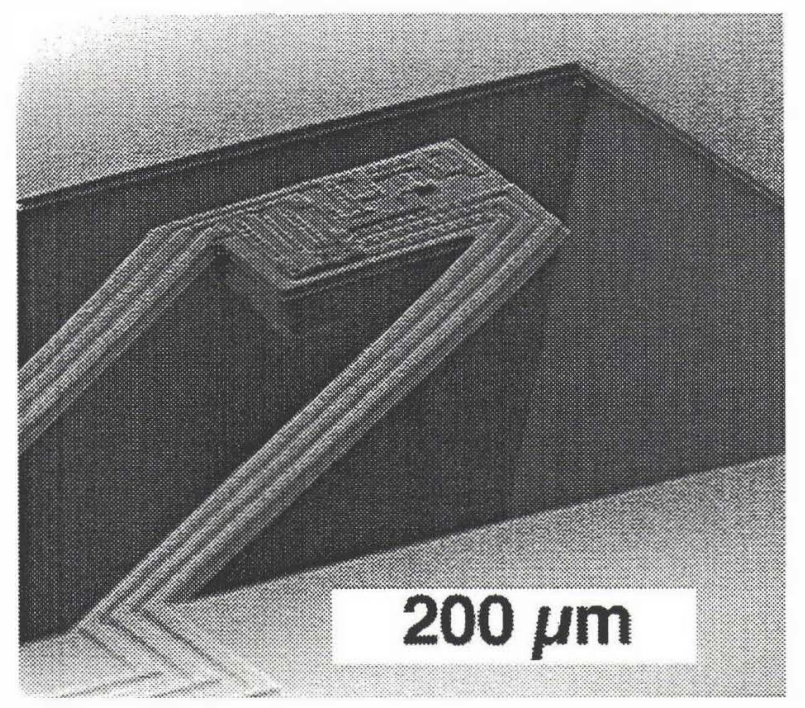

Figure 9. SEM of an etched vacuum sensor element.

Figure 10 shows the output of the sensor system for a device with $340 \mu \mathrm{m}$ long beams, for different operating temperatures. The pressure was measured with an MKS Baratron, a bellows-type capacitive vacuum sensor. As the graph shows, the effective sensitivity is larger for higher operating temperatures, since all the points on the curve scale with the setpoint. The output is the current $(\mu \mathrm{A})$ through the PMOS heater for a fixed source-drain voltage of $3 \mathrm{~V}$. For this sensor, the ratio of the thermal resistance in air to that at low vacuum was approximately 5.9 . 
Output (uW)

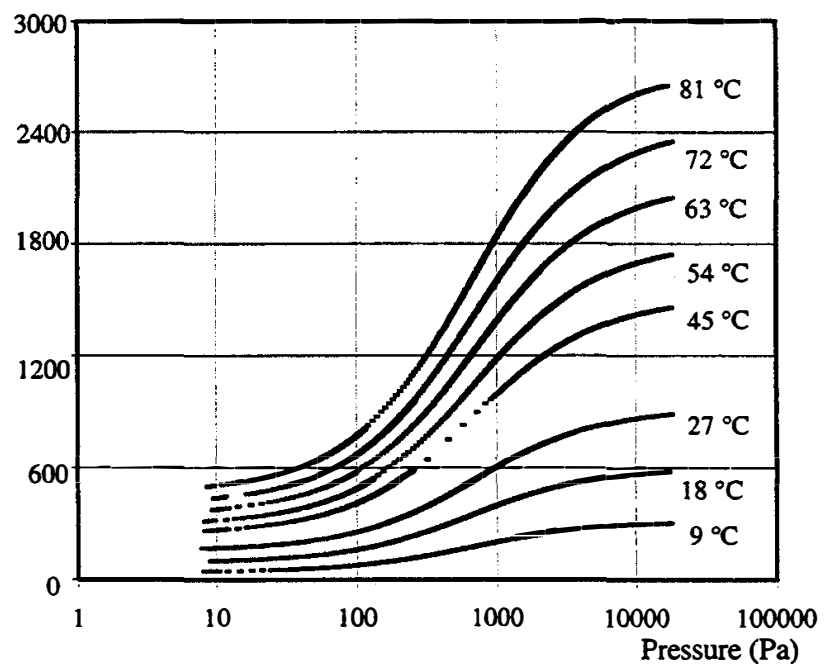

Figure 10. Sensor output over pressure for various operating temperatures relative to the substrate.

Figure 11 shows the performance of the sensor over its entire pressure range. The pressure is measured with an MKS Baratron below $1 \times 10^{4} \mathrm{~Pa}$ and with a Hastings 760 Thermocouple gauge for pressures above this point. The output is plotted as

$$
I_{N}=\frac{I(P)-I_{L P}}{I_{F S}}
$$

where $l(P)$ is the current at a given pressure, $I_{L P}$ is the current at low pressure ( $0.1 \mathrm{mTor})$, below the range of the sensor, and $I_{F S}$ is the current at ambient [2]. This plot is for a set-point of $90^{\circ} \mathrm{C}$ above the substrate temperature. The vacuum sensor has a range of $0.8 \mathrm{~Pa}(6 \mathrm{mTor})$ to approximately $9.2 \times 10^{4} \mathrm{~Pa}(690$ Torr).

The entire system occupies less than $0.3 \mathrm{~mm}^{2}$ and consumes approximately $5 \mathrm{~mW}$ of power (for a setpoint of $90^{\circ} \mathrm{C}$ above the substrate temperature).

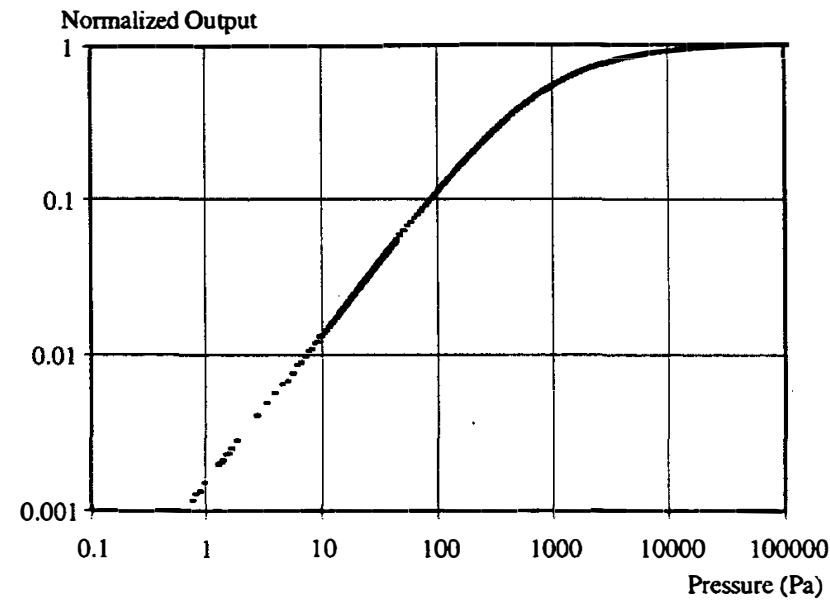

Figure 11. Plot of normalized sensor output over pressure.

\section{CONCLUSIONS AND OUTLOOK}

The fully integrated vacuum system presented here has a useful pressure range of more than five decades. It was built using an unmodified foundry CMOS process, which makes relatively inexpensive fabrication possible. The device could be further improved by providing some type of cover close to the suspended element [2]. This would increase the conductive losses through the air relative to that through the beams. Another possible improvement is to fabricate the device on wafers containing a buried oxide layer. This buried oxide layer would serve as an etch-stop during the undercutting etch, and would limit the depth of the anisotropically etched cavity.

\section{REFERENCES}

1. L. Carpenter, Vacuum Technology, An Introduction, American Elsevier, New York, 1970, pp. 49-55.

2. A. van Herwaarden, "Thermal vacuum sensors based on integrated silicon thermopiles," Ph.D. Thesis, Delft University of Technology, 1987.

3. C.H. Mastrangelo and R.S. Muller, "Fabrication and Performance of a Fully Integrated $\mu$-Pirani Pressure Gauge with Digital Readout," In Proceedings of Transducers '91, San Francisco, pp. 245-248, 1991.

4. S.D. James, R.G. Johnson, and R.E. Higashi, "A Broad Range Absolute Pressure Microsensor" In Technical Digest, IEEE Solid State Sensor and Actuator Workshop, Hilton Head, pp. 107-108, 1988.

5. O. Paul, A. Haeberli, P. Malcovati, and H. Baltes, "Novel Integrated Thermal Pressure Gauge and Read-Out Circuit by CMOS IC Technology," In Technical Digest, IEEE International Electron Devices Meeting, San Francisco, pp. 131-134, 1994.

6. N.R. Swart and A. Nathan, "An Integrated CMOS Polysilicon Coil-Based Micro-Pirani Gauge with High Heat Transfer Efficiency," In Technical Digest, IEEE Intermational Electron Devices Meeting, San Francisco, pp. 135-138, 1994.

7. U. Bonne and D. Kubisiak, "Burstproof, thermal pressure sensor for gases," in Technical Digest, Solid State Sensor and Actuator Workshop, held June 13-16, 1994, Hilton Head Island, SC, pp. 78-81.

8. Mosis $2.0 \mu \mathrm{m}$ Analog CMOS process. 4676 Admiralty Way, Marina del Rey, CA 90292-6695.

9. R. J. Reay, E. H. Klaassen, G. T. A. Kovacs, "Thermally and Electrically Isolated Single Crystal Silicon Structures in CMOS Technology," IEEE Electron Device Letters, no. 15, pp. 399-401, 1994.

10. E. H. Klaassen, R. J. Reay, C. Storment, J. Audy, P. Henry, A. P. Brokaw, G. T. A. Kovacs, "Micromachined Thermally Isolated Circuits," in Technical Digest, Solid State Sensor and Actuator Workshop, 1996, Hilton Head Island, SC. 